\title{
Stability of a Circular Epitaxial Island *
}

\author{
Bo $\mathrm{Li}^{\dagger} \quad$ Andreas Rätz ${ }^{\ddagger} \quad$ Axel Voigt ${ }^{\S}$
}

August 27, 2004

\begin{abstract}
The morphological stability of a single, epitaxially growing, circular adatom island with a radially symmetric adatom distribution is studied using a BurtonCabrera-Frank type island dynamics model. Various kinds of boundary conditions for the adatom density that include the thermodynamic equilibrium value, line tension, and attachment-detachment kinetics, and different velocity formulas with or without the one-dimensional "surface" diffusion are examined. Rigorous analysis shows that the circular island is always stable if its normalized area $A$ is larger than a critical value. If $A$ is less than such a critical value, and if neither the line tension nor surface diffusion is present, then there exists a critical wavenumber $k_{c}=k_{c}(A)$ such that the island is only stable for wavenumbers less than $k_{c}$. When the line tension or surface diffusion is present, small islands are always stable. In particular, the Bales-Zangwill instability for straight steps due to the kinetic asymmetry does not exist for small circular islands.
\end{abstract}

PACS numbers: 68.35.J (Surface dynamics); 68.55.a (Thin film structure and morphology); 81.10.Aj (Theory and models of crystal growth).

Keywords: epitaxial growth, island dynamics, morphological stability, line tension, surface diffusion, attachment-detachment kinetics, circular islands.

\section{Introduction}

The morphology of an epitaxially growing thin film surface consists of atomic terraces or islands, steps or island boundaries, and kinks, cf. Figure 1, and is determined by the

*This work was partially supported by DFG through SFB 611 and NSF through DMS-0072958.

$\dagger$ Department of Mathematics, University of California, San Diego, 9500 Gilman Drive, La Jolla, CA 92093-0112, USA. E-mail: bli@math.ucsd.edu.

${ }_{\ddagger}^{\ddagger}$ Crystal Growth Group, Research Center caesar, Ludwig-Erhard-Allee 2, D-53175, Bonn, Germany. E-mail: raetz@caesar.de.

${ }^{\S}$ Crystal Growth Group, Research Center caesar, Ludwig-Erhard-Allee 2, D-53175, Bonn, Germany. E-mail: voigt@caesar.de. 
interplay between the microscopic processes such as atom adsorption and desorption, atomic island nucleation, adatom (adsorbed atoms) diffusion, adatom attachment and detachment to and from island boundaries, and island coalescence $[4,14,18]$.

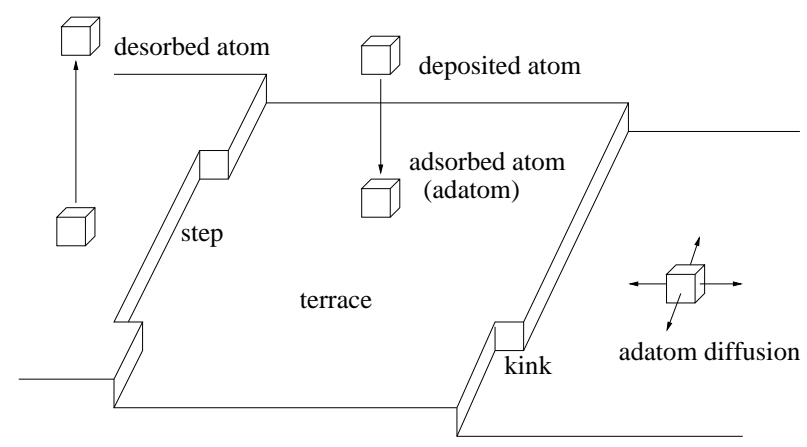

Figure 1: Microscopic processes in epitaxial growth of thin films.

Burton, Cabrera, and Frank [5] first developed a systematic and detailed modelBCF model - to describe the adatom density and the motion of steps in epitaxial growth of thin films. In this model, the adatom density solves a diffusion equation with an equilibrium boundary condition, and steps move at a velocity determined from a twosided diffusive flux of adatoms to the edges. Modifications of the BCF model have been made in $[3,10,11,13,15]$ to incorporate into the boundary conditions additional effects, such as the curvature of the step or boundary and in particular the Ehrlich-Schwoebel barrier - a higher energy barrier that must be overcome by an adatom in order for it to stick to the boundary from an upper terrace [8, 20, 21], cf. Figure 2. Such asymmetry in the attachment and detachment of adatoms to and from terrace boundaries is the origin of the Bales-Zangwill morphological instability for straight atomic steps [3]. Caflisch et al [6] have recently developed a kinetic step model that includes the kinetics missing in the original BCF model. Based on such a kinetic model, Caflisch and Li [7] have derived rigorously a set of boundary conditions for the adatom density that includes line tension and attachment-detachment kinetics, and a normal velocity law that includes one-dimensional "surface" diffusion, cf. also [12, 14, 17, 18, 19].

In this work, we use a BCF type island dynamics model to study the morphological stability of a single, epitaxially growing, circular adatom island with a radially symmetric adatom distribution with respect to perturbation in both radial and angular directions. This problem is of practical interest, since in the early stage of epitaxial growth, adatom islands are often small and circularly shaped, and are also far apart from each other so that their interaction can be neglected [16, 22, 23]. Similarly, in the coarsening process of epitaxial growth, shrinking islands are often small and circularly shaped, and their interaction with other islands, mainly through a mean field, can be weak [16, 22].

We assume that the growing circular island is contained geometrically in a large concentric circle on the surface. On the outer boundary of this large circle, we impose the 


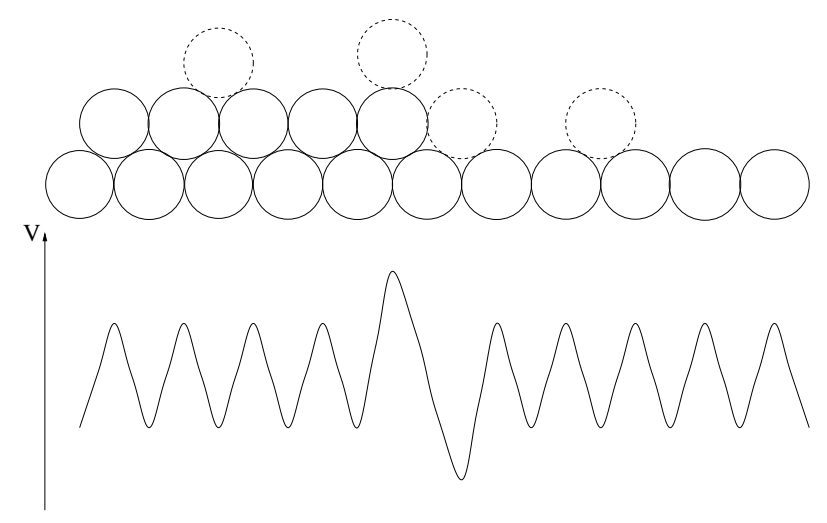

Figure 2: The Ehrlich-Schwoebel barrier.

flux-free boundary condition. This models the larger circle as the capture zone of the growing circular adatom island. We examine both thermodynamic and kinetic boundary conditions and their related normal velocity laws. The thermodynamic boundary conditions are determined by the Gibbs-Thomson relation in terms of adatom equilibrium value and line tension. The kinetic boundary conditions include the attachment-detachment kinetics. The related normal velocity is determined by the two-sided flux, together with the one-dimensional "surface" diffusion. We remark that, unlike for the motion of a straight step in step-flow growth, there is no kinetic steady state with a constant normal velocity for a growing circular island.

Our main results are the following:

(1) The circular island is always stable if its normalized area $A$ is larger than a critical value;

(2) If the normalized area $A$ is less than this critical value, and if neither the line tension nor surface diffusion is present, then there exists a critical wavenumber $k_{c}=k_{c}(A)$ such that the island is only stable for wavenumbers less than $k_{c}$.

(3) If the line tension or surface diffusion is present, small circular islands are always stable. In particular, the small wavenumber Bales-Zangwill instability [3] for straight steps due to the Ehrlich-Schwoebel effect does not exist for small circular islands.

In $[1,2]$, a single disk on a substrate is considered in the special case of only one-sided attachment and by neglecting one-dimensional "surface" diffusion. Moreover, a constant mean concentration is used as a far-field condition. A disk-shaped ice crystal growing in undercooled water is considered in [9]. Our underlying physical problems and the related models differ from the previous ones in having an external flux (the deposition flux), a flux-free (instead of a mean-field density value) far-field condition, the kinetic attachment-detachment condition, and the one-dimensional "surface" diffusion. 


\section{Description of dynamics of a single epitaxial island}

Consider a single, epitaxially growing island on a large crystal surface. The island is one atomic layer higher than the neighboring surface. Denote by $\Omega_{+}(t)$ and $\Omega_{-}(t)$, respectively, the regions occupied by the island (the upper terrace) and that of the neighboring surface (the lower terrace) at time $t$. Denote also by $\Gamma(t)=\overline{\Omega_{+}(t)} \cap \overline{\Omega_{-}(t)}$ the boundary of the island at time $t$. In the framework of a BCF model, the dynamics of the single island is described by the diffusion equation for the adatom density $\rho=\rho(x, t)$ with $x=\left(x_{1}, x_{2}\right)$ on the surface except the island boundary $\Gamma(t)$, the boundary condition for $\rho$ on the boundary $\Gamma(t)$, and the normal velocity $v$ of the boundary $\Gamma(t)$. The diffusion equation is $[5,11,18]$

$$
\partial_{t} \rho-D \nabla^{2} \rho=F-\tau^{-1} \rho-N \quad \text { in } \Omega_{+}(t) \cup \Omega_{-}(t),
$$

where $D>0$ is the diffusion constant, $F>0$ the deposition flux rate which is assumed to be a constant, $\tau^{-1}$ the desorption rate, and $N$ the rate of island nucleation.

There are mainly two classes of boundary conditions.

A. Thermodynamic Boundary Conditions (TBC) [3, 5, 11, 14, 18, 19]:

$$
\rho=\rho_{e}(1+\gamma \kappa) \quad \text { on } \Gamma(t),
$$

where $\rho_{e}$ is a thermodynamic equilibrium value of the adatom density for a straight step, $\gamma \geq 0$ represents the step stiffness, and $\kappa$ is the curvature of the boundary $\Gamma(t)$. We adopt the convention that $\kappa>0$ for a convex curve such as a circle.

B. Kinetic Boundary Conditions (KBC) [3, 7, 11, 12, 14, 18, 19]:

$$
\left\{\begin{aligned}
-D \nabla \rho_{+} \cdot n & =k_{+}\left(\rho_{+}-\rho_{*}-\mu \kappa\right) \\
D \nabla \rho_{-} \cdot n & =k_{-}\left(\rho_{-}-\rho_{*}-\mu \kappa\right)
\end{aligned} \quad \text { on } \Gamma(t),\right.
$$

where $\rho_{+}$and $\rho_{-}$denote the restriction of the density $\rho$ onto the boundary $\Gamma(t)$ from the upper and lower terraces, respectively, $n$ is the unit normal of the boundary $\Gamma(t)$ pointing from the upper into lower terrace, $k_{+}$and $k_{-}$are the attachment rates of adatoms to the boundary $\Gamma(t)$ from the upper and lower terraces, respectively, $\rho_{*}$ is a reference density, and $\mu \geq 0$ is a constant. Since the normal velocity $v$ of the island boundary is small in a typical epitaxial growth, we drop in the boundary condition (2.3) the usual convection term $v \rho[7,11]$. The term $\rho_{*}$ can be either a thermodynamic equilibrium value or a kinetic steady state value. For simplicity, we shall take $\rho_{*}$ to be a constant. The kinetic constant $\mu$ can be proportional to the stiffness of the boundary $\Gamma(t)$ or can come from a transition energy barrier, see $[3,5,6,7,17,18]$. In general, we have $k_{+} \leq k_{-}$with our notation. The strict inequality models the Ehrlich-Schwoebel effect. Formally, setting $k_{-}=k_{+}=\infty$ in (2.3), we obtain (2.2) with $\rho_{e}=\rho_{*}$ and $\gamma=\mu / \rho_{*}$.

We assume the following law for the normal velocity $v$ of the island boundary $\Gamma(t)$ that is derived by Caflisch and $\mathrm{Li}[7]$ from kinetics (cf. also [14])

$$
v=-D[\nabla \rho \cdot n]+\nu \kappa_{s s},
$$


where the bracket $[\cdot]$ denotes the jump across the island boundary and is defined for any $u$ by $[u]:=\left.u\right|_{\Omega_{+}(t)}-\left.u\right|_{\Omega_{-}(t)}$ on $\Gamma(t), \nu \geq 0$ is a constant, and the subscript $s$ denotes the tangential derivative along the boundary. The term $\kappa_{s s}$ represents one-dimensional "surface" diffusion. The coefficient $\nu$ is related to line tension and edge diffusion [7].

Finally, we assume that both the upper and lower terraces are enclosed in a large, fixed circle $\Gamma_{\infty}$ with radius $R_{\infty}$. We impose the flux-free far-field condition

$$
\nabla \rho \cdot n=0 \quad \text { on } \Gamma_{\infty}
$$

where $n$ denotes the unit exterior normal at the $\Gamma_{\infty}$. Such a boundary condition models the large, circular region as the capture zone of the single island.

Under typical conditions for epitaxial growth, the diffusion constant is very large, the desorption is negligible, and the island growth rate is very small due to a low deposition rate. For simplicity, we will leave out the nucleation term $N$. Therefore, in what follows, we shall only consider, instead of (2.1), the quasi-steady diffusion equation

$$
-D \Delta \rho=F \quad \text { in } \Omega_{+}(t) \cup \Omega_{-}(t) .
$$

The dynamics is recovered through the motion of the island boundary $\Gamma(t)$.

In summary, our mathematical formulation of the underlying problem consists of the diffusion equation (2.6), the boundary condition for $\rho$ given by (2.2) or (2.3), the far-field condition (2.5), and the velocity law (2.4). In addition, the adatom density $\rho$ is assumed to be continuous at the center of the island.

\section{A single circular island and its perturbation}

We now take the single island described above to be a circular island centered at the origin $O$ with radius $R(t)$ at time $t$, cf. Figure 3. The upper terrace, i.e., the circular island, occupies $\Omega_{+}(t)$, and the lower terrace occupies $\Omega_{-}(t)$. The corresponding island boundary $\Gamma(t)$ is the circle $r=R(t)$ with $r=|x|$. The outer boundary of the entire

circular region $\overline{\Omega_{+}(t) \cup \Omega_{-}(t)}$, which is also centered at the origin $O$, is $\Gamma_{\infty}: r=R_{\infty}$. We assume that $0<R_{0} \ll R_{\infty}$ with $R_{0}=R(0)$. The unit normal $n$, the normal velocity $v$, and the curvature $\kappa$ of the circular boundary $\Gamma(t)$ are given by

$$
n=(\cos \theta, \sin \theta), \quad v=R^{\prime}(t), \quad \kappa=1 / R(t),
$$

respectively, where $\theta$ is the angle in the polar coordinate system.

\subsection{Radially symmetric solution}

We consider the radially symmetric solution $\rho=\rho(r, t)$ with $r=|x|$ for the problem

$$
-D \Delta \rho=F \quad \text { in } \Omega_{+}(t) \cup \Omega_{-}(t),
$$




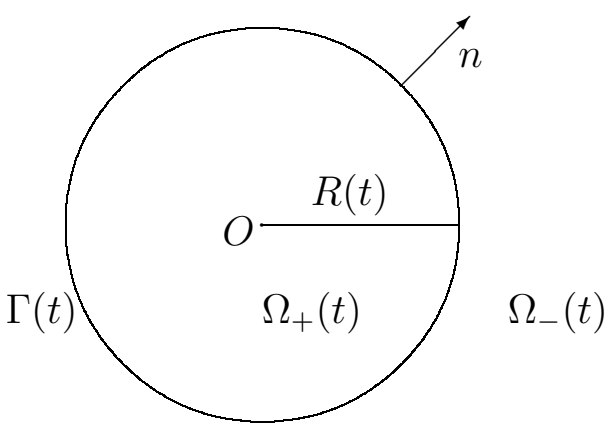

Figure 3: A circular epitaxial island.

$$
\begin{aligned}
& \text { boundary conditions for } \rho \text { on } \Gamma(t): r=R(t) \text {, } \\
& \rho \text { is continuous at } r=0 \text {, } \\
& \nabla \rho \cdot n=0 \quad \text { on } \Gamma_{\infty}: r=R_{\infty}, \\
& v=-D[\nabla \rho \cdot n]+\nu \kappa_{s s} \quad \text { on } \Gamma(t): r=R(t),
\end{aligned}
$$

where the boundary condition (3.3) is given by (2.2) or (2.3). Since $\Delta \rho=\frac{1}{r} \partial_{r}\left(r \rho_{r}\right)$ and the curvature $\kappa=1 / R(t)$ is spatially constant, we get from (3.2)-(3.6) that

$$
\begin{aligned}
& \rho(r, t)= \begin{cases}\rho_{+}(r, t)=\frac{F}{4 D}\left(R(t)^{2}-r^{2}\right)+C_{+}(t) & \text { in } \Omega_{+}(t), \\
\rho_{-}(r, t)=\frac{F}{4 D}\left(R(t)^{2}-r^{2}\right)+\frac{F R_{\infty}^{2}}{2 D} \ln \left(\frac{r}{R(t)}\right)+C_{-}(t) & \text { in } \Omega_{-}(t),\end{cases} \\
& \text { for TBC: } \quad C_{+}(t)=C_{-}(t)=\rho_{e}\left(1+\frac{\gamma}{R(t)}\right), \\
& \text { for KBC: }\left\{\begin{array}{l}
C_{+}(t)=\rho_{*}+\frac{\mu}{R(t)}+\frac{F R(t)}{2 k_{+}} \\
C_{-}(t)=\rho_{*}+\frac{\mu}{R(t)}+\frac{F}{2 k_{-}}\left(\frac{R_{\infty}^{2}}{R(t)}-R(t)\right),
\end{array}\right. \\
& v=F R_{\infty}^{2} /(2 R(t))=\left(F R_{\infty}^{2} / 2\right) \kappa .
\end{aligned}
$$

Since $v=R^{\prime}(t)$, the radius $R(t)$ satisfies $\left(R(t)^{2}\right)^{\prime}=F R_{\infty}^{2}$. Thus, with $R(0)=R_{0}$, we obtain the mass conservation

$$
A(t)=A_{0}+F t \quad \forall t>0,
$$

where $A(t)=\left(R(t) / R_{\infty}\right)^{2}$ is the normalized area and $A_{0}=\left(R(0) / R_{\infty}\right)^{2}$. Notice that there exists a unique $t_{\infty}>0$ defined by $R\left(t_{\infty}\right)=R_{\infty}$ that satisfies

$$
t_{\infty}=\frac{R_{\infty}^{2}-R_{0}^{2}}{F R_{\infty}^{2}}=\frac{1}{F}\left(1-A_{0}\right)<\frac{1}{F} .
$$




\subsection{Perturbation}

Consider the perturbation in both radial and angular directions

$$
\begin{aligned}
& \tilde{\rho}(r, \theta, t)=\rho(r, t)+\varepsilon \rho_{1}(r, \theta, t), \\
& \tilde{R}(\theta, t)=R(t)+\varepsilon R_{1}(\theta, t)
\end{aligned}
$$

where $\varepsilon$ is a parameter small in magnitude. Denote by $\tilde{\Gamma}(t)=\{r=\tilde{R}(\theta, t)\}$ the perturbed island boundary. Denote also by $\tilde{\Omega}_{+}(t)$ and $\tilde{\Omega}_{-}(t)$ the perturbed upper and lower terraces, respectively. The perturbed $\tilde{\rho}(r, \theta, t)$ and $\tilde{R}(\theta, t)$, together with the corresponding normal velocity $\tilde{v}$ and curvature $\tilde{\kappa}$, satisfy a set of equations, similar to (3.2)-(3.6), on $\tilde{\Omega}_{+}(t) \cup$ $\tilde{\Omega}_{-}(t)$. In particular, the boundary conditions are

$$
\begin{array}{ll}
\text { TBC: } & \tilde{\rho}=\rho_{e}(1+\gamma \tilde{\kappa}) \quad \text { on } \tilde{\Gamma}(t): r=\tilde{R}(\theta, t), \\
\mathrm{KBC}: \quad\left\{\begin{array}{c}
-D \nabla \tilde{\rho}_{+} \cdot \tilde{n}=k_{+}\left(\tilde{\rho}_{+}-\rho_{*}-\mu \tilde{\kappa}\right) \\
D \nabla \tilde{\rho}_{-} \cdot \tilde{n}=k_{-}\left(\tilde{\rho}_{-}-\rho_{*}-\mu \tilde{\kappa}\right)
\end{array} \quad \text { on } \tilde{\Gamma}(t): r=\tilde{R}(\theta, t),\right.
\end{array}
$$

and the normal velocity is

$$
\tilde{v}=-D[\nabla \tilde{\rho} \cdot \tilde{n}]+\nu \tilde{\kappa}_{\tilde{s} \tilde{s}} \quad \text { on } \tilde{\Gamma}(t): r=\tilde{R}(\theta, t)
$$

Standard calculations lead to the following linearized system for the perturbation $\rho_{1}=\rho_{1}(r, \theta, t)$ and $R_{1}=R_{1}(\theta, t)$, in which the boundary conditions (3.22) and (3.23) and the velocity law (3.21) are derived in Appendix:

$$
\begin{aligned}
& \Delta \rho_{1}=0 \quad \text { in } \Omega_{+}(t) \cup \Omega_{-}(t), \\
& \text { boundary conditions for } \rho_{1} \text { on } \Gamma(t): r=R(t), \\
& \rho_{1} \text { is continuous at } r=0, \\
& \partial_{r} \rho_{1}\left(R_{\infty}, \theta, t\right)=0, \\
& \partial_{t} R_{1}(\theta, t)=-D\left[R_{1}(\theta, t) \partial_{r r} \rho(R(t), t)+\partial_{r} \rho_{1}(R(t), \theta, t)\right] \\
& \quad-\frac{\nu}{(R(t))^{4}}\left(\partial_{\theta \theta \theta \theta} R_{1}(\theta, t)+\partial_{\theta \theta} R_{1}(\theta, t)\right),
\end{aligned}
$$

where the boundary conditions (3.18) are given by

$$
\mathrm{TBC}:\left\{\begin{array}{l}
R_{1}(\theta, t) \partial_{r} \rho_{+}(R(t), t)+\rho_{1+}(R(t), \theta, t)=-\frac{\rho_{e} \gamma}{(R(t))^{2}}\left(\partial_{\theta \theta} R_{1}(\theta, t)+R_{1}(\theta, t)\right), \\
R_{1}(\theta, t) \partial_{r} \rho_{-}(R(t), t)+\rho_{1-}(R(t), \theta, t)=-\frac{\rho_{e} \gamma}{(R(t))^{2}}\left(\partial_{\theta \theta} R_{1}(\theta, t)+R_{1}(\theta, t)\right),
\end{array}\right.
$$


$\mathrm{KBC}:\left\{\begin{array}{r}-D R_{1}(\theta, t) \partial_{r r} \rho_{+}(R(t), t)-D \partial_{r} \rho_{1+}(R(t), \theta, t)=k_{+}\left(R_{1}(\theta, t) \partial_{r} \rho_{+}(R(t), t)\right. \\ \left.+\rho_{1+}(R(t), \theta, t)+\frac{\mu}{(R(t))^{2}}\left(\partial_{\theta \theta} R_{1}(\theta, t)+R_{1}(\theta, t)\right)\right) \\ D R_{1}(\theta, t) \partial_{r r} \rho_{-}(R(t), t)+D \partial_{r} \rho_{1-}(R(t), \theta, t)=k_{-}\left(R_{1}(\theta, t) \partial_{r} \rho_{-}(R(t), t)\right. \\ \left.+\rho_{1-}(R(t), \theta, t)+\frac{\mu}{(R(t))^{2}}\left(\partial_{\theta \theta} R_{1}(\theta, t)+R_{1}(\theta, t)\right)\right) .\end{array}\right.$

\subsection{Dispersion relation}

Consider perturbations $R_{1}=R_{1}(\theta, t)$ and $\rho_{1}=\rho_{1}(r, \theta, t)$ which are periodic in the angular variable $\theta$. The general solution of the linearized system (3.17)-(3.21) is of the form

$$
\begin{aligned}
R_{1}(\theta, t) & =\sum_{k=-\infty}^{+\infty} \hat{R}_{1, k} e^{\omega(k, t)} e^{i k \theta}, \\
\rho_{1}(r, \theta, t) & = \begin{cases}\sum_{k=-\infty}^{+\infty} U_{1+}(k, r, t) e^{\omega(k, t)+i k \theta} & \text { in } \Omega_{+}(t), \\
\sum_{k=-\infty}^{+\infty} U_{1-}(k, r, t) e^{\omega(k, t)+i k \theta} & \text { in } \Omega_{-}(t),\end{cases}
\end{aligned}
$$

where, for each integer wavenumber $k \geq 0, \hat{R}_{1, k}$ is a constant, $\omega(k, t)$ is the growth exponent whose time derivative $\partial_{t} \omega(k, t)$ represents the growth rate, $i=\sqrt{-1}$, and $U_{1+}(k, r, t)$ and $U_{1-}(k, r, t)$ are independent of $\theta$. To study the linearized stability, however, it suffices for us to consider the principal mode solution

$$
\begin{aligned}
& R_{1, k}(\theta, t)=\hat{R}_{1, k} e^{\omega(k, t)+i k \theta}, \\
& \rho_{1, k}(r, \theta, t)= \begin{cases}U_{+}(k, r, t) e^{\omega(k, t)+i k \theta} & \text { in } \Omega_{+}(t), \\
U_{-}(k, r, t) e^{\omega(k, t)+i k \theta} & \text { in } \Omega_{-}(t),\end{cases}
\end{aligned}
$$

for any integer wave number $k \geq 0$.

Since the Laplacian in the polar coordinate system is given by $\Delta=\partial_{r r}+(1 / r) \partial_{r}+$ $\left(1 / r^{2}\right) \partial_{\theta \theta}$, we have by (3.17) that $U_{r r}+(1 / r) U_{r}-\left(k^{2} /\left(r^{2}\right) U=0\right.$, where $U=U_{1 \pm}$. Set $U=r^{\alpha}$ to get $\alpha(\alpha-1)+\alpha-k^{2}=0$, i.e., $\alpha= \pm k$. Thus, if $k>0$,

$$
\rho_{1, k}(r, \theta, t)= \begin{cases}\left(\hat{a}_{+} r^{k}+\hat{b}_{+} r^{-k}\right) e^{\omega(k, t)+i k \theta} & \text { in } \Omega_{+}(t), \\ \left(\hat{a}_{-} r^{k}+\hat{b}_{-} r^{-k}\right) e^{\omega(k, t)+i k \theta} & \text { in } \Omega_{-}(t),\end{cases}
$$


where $\hat{a}_{ \pm}=\hat{a}_{ \pm}(k, t)$ and $\hat{b}_{ \pm}=\hat{b}_{ \pm}(k, t)$ are independent of $\theta$ and $r$. By (3.19) and (3.20), we get $\hat{b}_{+}=0$ and $\hat{b}_{-}=R_{\infty}^{2 k} \hat{a}_{-}$. Therefore,

$$
\rho_{1, k}(r, \theta, t)= \begin{cases}\hat{a}_{+} r^{k} e^{\omega(k, t)+i k \theta} & \text { in } \Omega_{+}(t), \\ \hat{a}_{-}\left(r^{k}+R_{\infty}^{2 k} r^{-k}\right) e^{\omega(k, t)+i k \theta} & \text { in } \Omega_{-}(t) .\end{cases}
$$

This is also true for $k=0$.

By a series of calculations, we obtain from the TBC (3.22) that

$$
\begin{aligned}
& (R(t))^{k} \hat{a}_{+}+\left(\frac{\rho_{e} \gamma\left(1-k^{2}\right)}{(R(t))^{2}}-\frac{F R(t)}{2 D}\right) \hat{R}_{1, k}=0, \\
& \left((R(t))^{k}+\frac{R_{\infty}^{2 k}}{(R(t))^{k}}\right) \hat{a}_{-}+\left(\frac{\rho_{e} \gamma\left(1-k^{2}\right)}{(R(t))^{2}}-\frac{F R(t)}{2 D}+\frac{F R_{\infty}^{2}}{2 D R(t)}\right) \hat{R}_{1, k}=0,
\end{aligned}
$$

from the $\mathrm{KBC}(3.23)$ that

$$
\begin{aligned}
& -\left(D k(R(t))^{k-1}+k_{+}(R(t))^{k}\right) \hat{a}_{+}+\left(\frac{F}{2}+\frac{k_{+} F R(t)}{2 D}-\frac{k_{+} \mu\left(1-k^{2}\right)}{(R(t))^{2}}\right) \hat{R}_{1, k}=0, \\
& \left\{D k\left((R(t))^{k-1}-\frac{R_{\infty}^{2 k}}{(R(t))^{k+1}}\right)-k_{-}\left((R(t))^{k}+\frac{R_{\infty}^{2 k}}{(R(t))^{k}}\right)\right\} \hat{a}_{-} \\
& \quad+\left\{-\frac{F}{2}\left(1+\frac{R_{\infty}^{2}}{(R(t))^{2}}\right)+\frac{k_{-} F}{2 D}\left(R(t)-\frac{R_{\infty}^{2}}{R(t)}\right)-\frac{k_{-} \mu\left(1-k^{2}\right)}{(R(t))^{2}}\right\} \hat{R}_{1, k}=0,
\end{aligned}
$$

and from the velocity formula (3.21) that

$$
\begin{aligned}
& D k(R(t))^{k-1} \hat{a}_{+}-D k\left((R(t))^{k-1}-\frac{R_{\infty}^{2 k}}{(R(t))^{k+1}}\right) \hat{a}_{-} \\
& \quad+\left(\partial_{t} \omega(k, t)+\frac{F R_{\infty}^{2}}{2(R(t))^{2}}+\frac{\nu\left(k^{4}-k^{2}\right)}{(R(t))^{4}}\right) \hat{R}_{1, k}=0 .
\end{aligned}
$$

In order to have a nontrivial solution (3.24) and (3.25), we must have $\hat{a}_{+}, \hat{a}_{-}$, and $\hat{R}_{1, k}$ not all zero. Thus, the homogeneous system of linear equations for $\hat{a}_{+}, \hat{a}_{-}$, and $\hat{R}_{1, k}$ must be singular. Consequently, for the TBC, we have from (3.27), (3.28), and (3.31) that

$$
\left|\begin{array}{ccc}
(R(t))^{k} & 0 & \frac{\rho_{e} \gamma\left(1-k^{2}\right)}{(R(t))^{2}}-\frac{F R(t)}{2 D} \\
0 & (R(t))^{k}+\frac{R_{\infty}^{2 k}}{(R(t))^{k}} & \frac{\rho_{e} \gamma\left(1-k^{2}\right)}{(R(t))^{2}}-\frac{F R(t)}{2 D}+\frac{F R_{\infty}^{2}}{2 D R(t)} \\
D k(R(t))^{k-1} & -D k\left((R(t))^{k-1}-\frac{R_{\infty}^{2 k}}{(R(t))^{k+1}}\right) & \partial_{t} \omega(k, t)+\frac{F R_{\infty}^{2}}{2(R(t))^{2}}+\frac{\nu\left(k^{4}-k^{2}\right)}{(R(t))^{4}}
\end{array}\right|=0 .
$$

Similarly, for the KBC, we have from (3.29)-(3.31) that

$$
\left|\begin{array}{ccc}
-D k R^{k-1}-k_{+} R^{k} & 0 & \frac{F}{2}+\frac{k_{+} F R}{2 D}-k_{+} P \\
0 & D k Q-k_{-}\left(R^{k}+\frac{R_{\infty}^{2 k}}{R^{k}}\right) & -\frac{F}{2}\left(1+\frac{R_{\infty}^{2}}{R^{2}}\right)+\frac{k_{-} F}{2 D}\left(R-\frac{R_{\infty}^{2}}{R}\right)-k_{-} P \\
D k R^{k-1} & -D k Q & \partial_{t} \omega(k, t)+\frac{F R_{\infty}^{2}}{2 R^{2}}+\frac{\nu\left(k^{4}-k^{2}\right)}{R^{4}}
\end{array}\right|=0,
$$


where

$$
R=R(t), \quad P=\frac{\mu\left(1-k^{2}\right)}{R^{2}}, \quad Q=R^{k-1}-\frac{R_{\infty}^{2 k}}{R^{k+1}} .
$$

These equations determine the dispersion relation, i.e., the growth rate $\partial_{t} \omega(k, t)$, for $k>0$. We say the circular island (linearly) stable at time $t \in\left[0, t_{\infty}\right)$ with respect to a wavenumber $k \geq 0$, if $\partial_{t} \omega(k, t)<0$, where $t_{\infty}$ is defined by $R\left(t_{\infty}\right)=R_{\infty}$, cf. (3.11).

\section{Stability analysis: thermodynamic boundary con- ditions}

Consider the thermodynamic boundary condition (3.14) and the general velocity (3.16). The definition of the normalized area $A=A(t)=\left(R(t) / R_{\infty}\right)^{2}$ leads to $R=R(t)=$ $R_{\infty} A^{1 / 2}$. Note $A_{0}=A(0)$. From (3.32), we obtain for all $k \geq 0$ that

$$
\partial_{t} \omega(k, t)=\frac{F\left((1-2 A) k-(k+1) A^{k}-1\right)}{2 A\left(1+A^{k}\right)}-\frac{2 D \rho_{e} \gamma\left(k^{3}-k\right)}{R_{\infty}^{3} A^{3 / 2}\left(1+A^{k}\right)}-\frac{\nu\left(k^{4}-k^{2}\right)}{R_{\infty}^{4} A^{2}} .
$$

Recall that we only consider wavenumber $k \geq 0$ which is an integer. The following results are true whether the line tension or surface diffusion is included or not.

Proposition 4.1 Let $\gamma \geq 0$ and $\nu \geq 0$.

(1) If $k=0,1$, then $\partial_{t} \omega(k, t)<0$ for any $t \in\left[0, t_{\infty}\right)$.

(2) Let $t \in\left[0, t_{\infty}\right)$ be such that $A=A(t) \geq 1 / 2$, then $\partial_{t} \omega(k, t)<0$ for any wavenumber $k$.

(3) We have for any $t \in\left[0, t_{\infty}\right)$ that

$$
\begin{aligned}
\partial_{t} \omega(k, t)=- & \frac{\nu}{R_{\infty}^{4} A^{2}} k^{4}-\frac{2 D \rho_{e} \gamma}{R_{\infty}^{3} A^{3 / 2}} k^{3}+\frac{\nu}{R_{\infty}^{4} A^{2}} k^{2} \\
& +\left(\frac{2 D \rho_{e} \gamma}{R_{\infty}^{3} A^{3 / 2}}+\frac{F(1-2 A)}{2 A}\right) k+O(1) \quad \text { as } k \rightarrow \infty .
\end{aligned}
$$

Proof. (1) By (4.1), $\partial_{t} \omega(0, t)=-F /(2 A)<0$ and $\partial_{t} \omega(1, t)=-2 F /(1+A)<0$.

(2) If $A \geq 1 / 2$, then by (4.1)

$$
\partial_{t} \omega(k, t) \leq-\frac{F\left((k+1) A^{k}+1\right)}{2 A\left(1+A^{k}\right)}<0 .
$$

(3) This follows from (4.1) and a series of straight forward calculations.

Q.E.D.

Proposition 4.2 Let $t \in\left[0, t_{\infty}\right)$. 
(1) Let $\gamma>0$ and $\nu \geq 0$. We have $\partial_{t} \omega(k, t)<0$ for any wavenumber $k$, if

$$
A(t) \leq\left(\frac{24 D \rho_{e} \gamma}{F R_{\infty}^{3}}\right)^{2}
$$

(2) Let $\gamma \geq 0$ and $\nu>0$. We have $\partial_{t} \omega(k, t)<0$ for all wavenumber $k$, if

$$
A(t) \leq \frac{24 \nu}{F R_{\infty}^{4}} .
$$

Proof. By Part (1) of Proposition 4.1, we may assume that $k \geq 2$.

(1) By (4.1) and (4.3), we have

$$
\partial_{t} \omega(k, t)<\frac{F(k-1)}{2 A\left(1+A^{k}\right)}-\frac{2 D \rho_{e} \gamma\left(k^{3}-k\right)}{R_{\infty}^{3} A^{3 / 2}\left(1+A^{k}\right)}=\frac{k-1}{A\left(1+A^{k}\right)}\left(\frac{F}{2}-\frac{12 D \rho_{e} \gamma}{R_{\infty}^{3} \sqrt{A}}\right) \leq 0 .
$$

(2) By (4.1) and (4.4), we have

$$
\partial_{t} \omega(k, t)<\frac{F(k-1)}{2 A}-\frac{\nu k^{2}\left(k^{2}-1\right)}{R_{\infty}^{4} A^{2}} \leq \frac{k-1}{A}\left(\frac{F}{2}-\frac{12 \nu}{R_{\infty}^{4} A^{2}}\right) \leq 0 .
$$

\section{Q.E.D.}

Proposition 4.3 Assume $\gamma=\nu=0$. Let $t \in\left[0, t_{\infty}\right)$ be such that $A_{0} \leq A=A(t)<1 / 2$. Then there exists a unique $k_{c}=k_{c}(A)>1 /(1-2 A)$ such that

$$
\partial_{t} \omega(k, t)<0 \quad \text { for } k<k_{c} \quad \text { and } \quad \partial_{t} \omega(k, t)>0 \quad \text { for } k>k_{c} .
$$

Proof. In this case, we have by (4.1) that

$$
\partial_{t} \omega(k, t)=\frac{F}{2 A\left(1+A^{k}\right)}\left((1-2 A) k-(k+1) A^{k}-1\right) .
$$

Define

$$
f(k):=(1-2 A) k-(k+1) A^{k}-1
$$

for any $k \geq 0$. By (4.6), $\partial_{t} \omega(k, t)=0$ if and only if $f(k)=0$. It is easy to see that $f(k)$ is smooth on $[0, \infty), f(k) \leq-(k+1) A^{k}<0$ for any $k \in[0,1 /(1-2 A)]$, and $\lim _{k \rightarrow \infty} f(k)=\infty$. Moreover, for any $k>1 /(1-2 A)>1$,

$$
f^{\prime}(k)=1-2 A-A^{k}(1+(k+1) \ln A)>-A^{k}\left(1+2 \ln \frac{1}{2}\right)=A^{k}(\ln 4-1)>0 .
$$

Thus, there exists a unique $k_{c}=k_{c}(A)>1 /(1-2 A)$ that satisfies (4.5). Q.E.D. 
Figure 4 is the stability diagram in the $(A, k)$-plane for the thermodynamics boundary condition with $\gamma=\nu=0$ and $A<1 / 2$. The curve, $k=k_{c}(A)$, is obtained by solving the equation $\partial_{t} \omega(k, t)=0$ in which the growth rate $\partial_{t} \omega(k, t)$ is given by $(4.1)$ with $\gamma=\nu=0$.

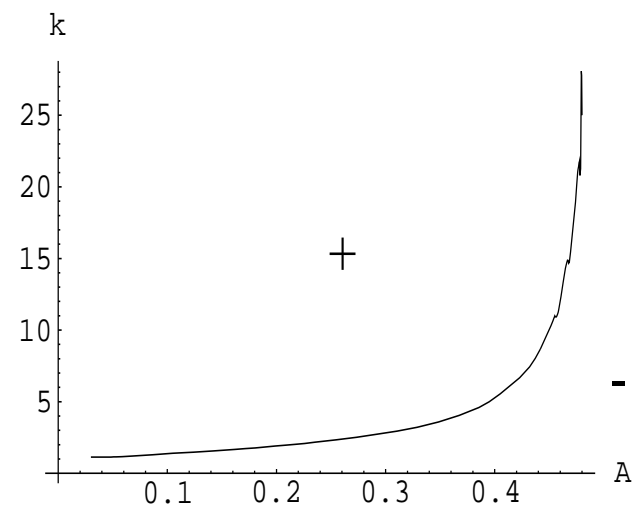

Figure 4: The stability diagram in the $(A, k)$-plane for the thermodynamic boundary condition with $\gamma=\nu=0$. The curve, $k=k_{c}(A)$, separates the stable region (marked by "-") and the unstable region (marked by "+").

We see from our analysis that the circular island is always stable if its normalized area $A \geq 1 / 2$. The island is asymptotically stable, with a decay rate proportional to $k^{4}$ if the surface diffusion is included, or to $k^{3}$ if the surface diffusion is not but the line tension is included. In general, a small circular island is always stable, if the line tension or surface diffusion is included. If $A<1 / 2$, and if neither the line tension nor surface diffusion is included, then there exists a critical wavenumber $k_{c}=k_{c}(A)$ such that the island is stable only for the wavenumber $k<k_{c}$.

\section{Stability analysis: kinetic boundary conditions}

Consider now the kinetic boundary condition (3.15) and the general velocity (3.16). From (3.33), we obtain using the fact that $R=R_{\infty} A^{1 / 2}$ that for all $k \geq 0$,

$$
\begin{aligned}
\partial_{t} \omega(k, t)=- & \frac{F}{2 A}-\frac{\nu\left(k^{4}-k^{2}\right)}{R_{\infty}^{4} A^{2}}-\frac{D k\left(\frac{F}{2}+\frac{k_{+} F R_{\infty} A^{1 / 2}}{2 D}+\frac{k_{+} \mu\left(k^{2}-1\right)}{R_{\infty}^{2} A}\right)}{D k+k_{+} R_{\infty} A^{1 / 2}} \\
& +\frac{D k\left(1-A^{k}\right)\left(\frac{F}{2}\left(1+\frac{1}{A}\right)+\frac{k_{-} F R_{\infty} A^{1 / 2}}{2 D}\left(\frac{1}{A}-1\right)-\frac{k_{-} \mu\left(k^{2}-1\right)}{R_{\infty}^{2} A}\right)}{k_{-} R_{\infty} A^{1 / 2}\left(A^{k}+1\right)+D k\left(1-A^{k}\right)} .
\end{aligned}
$$

For $k_{ \pm} \rightarrow \infty$, we recover (4.1) with $\rho_{e} \gamma=\mu$, as expected.

Proposition 5.1 Let $\mu \geq 0$ and $\nu \geq 0$.

(1) We have for any $t \in\left[0, t_{\infty}\right)$ and $k=0,1$ that $\partial_{t} \omega(k, t)<0$. 
(2) Let $t \in\left[0, t_{\infty}\right)$ be such that $A=A(t) \geq k_{-} /\left(k_{-}+k_{+}\right)$, then $\partial_{t} \omega(k, t)<0$ for any wavenumber $k$.

(3) For any $t \in\left[0, t_{\infty}\right)$, we have

$$
\begin{aligned}
\partial_{t} \omega(k, t)=- & \frac{\nu}{R_{\infty}^{4} A^{2}} k^{4}+\left(\frac{\nu}{R_{\infty}^{4} A^{2}}-\frac{\mu\left(k_{+}+k_{-}\right)}{R_{\infty}^{2} A}\right) k^{2}+\frac{\mu\left(k_{+}^{2}+k_{-}^{2}\right)}{D R_{\infty} A^{1 / 2}} k \\
& +\frac{F R_{\infty} A^{1 / 2}}{2 D}\left(k_{-}\left(\frac{1}{A}-1\right)-k_{+}\right)+O\left(\frac{1}{k}\right) \quad \text { as } k \rightarrow \infty .
\end{aligned}
$$

Proof. (1) By (5.1), $\partial_{t} \omega(0, t)=-F /(2 A)<0$ and

$$
\begin{aligned}
\partial_{t} \omega(1, t) & =-\frac{F}{2 A}-\frac{D\left(\frac{F}{2}+\frac{k_{+} F R_{\infty} A^{1 / 2}}{2 D}\right)}{D+k_{+} R_{\infty} A^{1 / 2}}+\frac{D(1-A)\left(\frac{F}{2}\left(1+\frac{1}{A}\right)+\frac{k_{-} F R_{\infty} A^{1 / 2}}{2 D}\left(\frac{1}{A}-1\right)\right)}{k_{-} R_{\infty} A^{1 / 2}(A+1)+D(1-A)} \\
& =-\frac{F}{2 A}-\frac{F}{2}+\frac{F}{2 A} \frac{D\left(1-A^{2}\right)+k_{-} R_{\infty} A^{1 / 2}(1-A)^{2}}{k_{-} R_{\infty} A^{1 / 2}(A+1)+D(1-A)} \\
& =\frac{F}{2 A} \frac{k_{-} R_{\infty} A^{1 / 2}\left((1-A)^{2}-(1+A)^{2}\right)}{k_{-} R_{\infty} A^{1 / 2}(A+1)+D(1-A)} \\
& =\frac{-2 F k_{-} R_{\infty} A^{1 / 2}}{k_{-} R_{\infty} A^{1 / 2}(A+1)+D(1-A)} \\
& <0 .
\end{aligned}
$$

(2) Since $k_{+} \leq k_{-}$and $A_{0} \leq A<1$, we have

$$
\frac{D k\left(1-A^{k}\right)}{D k\left(1-A^{k}\right)+k_{-} R_{\infty} A^{1 / 2}\left(1+A^{k}\right)} \leq \frac{D k\left(1-A^{k}\right)}{D k\left(1-A^{k}\right)+k_{+} R_{\infty} A^{1 / 2}} \leq \frac{D k}{D k+k_{+} R_{\infty} A^{1 / 2}},
$$

where in the second step the inequality $x /(x+z) \leq y /(y+z)$ for $x \leq y$ and $x, y, z>0$ is used. By (5.1), we get

$$
\begin{aligned}
\partial_{t} \omega(k, t) \leq- & \frac{F}{2 A}-\frac{D k\left(\frac{F}{2}+\frac{k_{+} F R_{\infty} A^{1 / 2}}{2 D}\right)}{D k+k_{+} R_{\infty} A^{1 / 2}} \\
& +\frac{D k\left(A^{k}-1\right)\left(-\frac{F}{2}\left(1+\frac{1}{A}\right)+\frac{k_{-} F R_{\infty} A^{1 / 2}}{2 D}\left(1-\frac{1}{A}\right)\right)}{k_{-} R_{\infty} A^{1 / 2}\left(A^{k}+1\right)-D k\left(A^{k}-1\right)} \\
=- & \frac{F}{2 A}-\frac{D k\left(\frac{F}{2}\right)}{D k+k_{+} R_{\infty} A^{1 / 2}}-\frac{D k\left(\frac{k_{+} F R_{\infty} A^{1 / 2}}{2 D}\right)}{D k+k_{+} R_{\infty} A^{1 / 2}} \\
& +\frac{D k\left(1-A^{k}\right)\left(\frac{F}{2}\left(1+\frac{1}{A}\right)\right)}{k_{-} R_{\infty} A^{1 / 2}\left(1+A^{k}\right)+D k\left(1-A^{k}\right)}+\frac{D k\left(1-A^{k}\right)\left(\frac{k_{-} F R_{\infty} A^{1 / 2}}{2 D}\right)\left(\frac{1}{A}-1\right)}{k_{-} R_{\infty} A^{1 / 2}\left(1+A^{k}\right)+D k\left(1-A^{k}\right)}
\end{aligned}
$$




$$
\begin{aligned}
\leq- & \frac{F}{2 A}-\frac{D k\left(\frac{F}{2}\right)}{D k+k_{+} R_{\infty} A^{1 / 2}}-\frac{D k\left(\frac{k_{+} F R_{\infty} A^{1 / 2}}{2 D}\right)}{D k+k_{+} R_{\infty} A^{1 / 2}} \\
& +\frac{D k\left(\frac{F}{2}\left(1+\frac{1}{A}\right)\right)}{D k+k_{+} R_{\infty} A^{1 / 2}}+\frac{D k\left(1-A^{k}\right)\left(\frac{k_{-} F R_{\infty} A^{1 / 2}}{2 D}\right)\left(\frac{1}{A}-1\right)}{k_{-} R_{\infty} A^{1 / 2}\left(1+A^{k}\right)+D k\left(1-A^{k}\right)} \\
=- & \frac{F}{2 A}+\frac{D k\left(\frac{F}{2 A}\right)}{D k+k_{+} R_{\infty} A^{1 / 2}}-\frac{D k\left(\frac{k_{+} F R_{\infty} A^{1 / 2}}{2 D}\right)}{D k+k_{+} R_{\infty} A^{1 / 2}} \\
& +\frac{D k\left(1-A^{k}\right)\left(\frac{k_{-} F R_{\infty} A^{1 / 2}}{2 D}\right)\left(\frac{1}{A}-1\right)}{k_{-} R_{\infty} A^{1 / 2}\left(1+A^{k}\right)+D k\left(1-A^{k}\right)} \\
\leq- & \frac{D k\left(\frac{k_{+} F R_{\infty} A^{1 / 2}}{2 D}\right)}{D k+k_{+} R_{\infty} A^{1 / 2}}+\frac{D k\left(1-A^{k}\right)\left(\frac{k_{-} F R_{\infty} A^{1 / 2}}{2 D}\right)\left(\frac{1}{A}-1\right)}{k_{-} R_{\infty} A^{1 / 2}\left(1+A^{k}\right)+D k\left(1-A^{k}\right)} .
\end{aligned}
$$

Consequently, a sufficient condition for $\partial_{t} \omega(k, t)<0$ in this case is that

$$
\begin{aligned}
& \frac{\left(1-A^{k}\right) k_{-}\left(\frac{1}{A}-1\right)}{k_{-} R_{\infty} A^{1 / 2}\left(A^{k}+1\right)+D k\left(1-A^{k}\right)}<\frac{k_{+}}{D k+k_{+} R_{\infty} A^{1 / 2}} \\
\Longleftrightarrow & \left(1-A^{k}\right) k_{-}\left(\frac{1}{A}-1\right)\left(D k+k_{+} R_{\infty} A^{1 / 2}\right)<k_{+}\left(k_{-} R_{\infty} A^{1 / 2}\left(A^{k}+1\right)+D k\left(1-A^{k}\right)\right) \\
\Longleftrightarrow & \frac{k_{-} D k\left(1-A^{k}\right)}{A}+\frac{k_{+} k_{-} R_{\infty}\left(1-A^{k}\right)}{A^{1 / 2}}<D k\left(1-A^{k}\right)\left(k_{-}+k_{+}\right)+2 k_{-} k_{+} R_{\infty} A^{1 / 2} .
\end{aligned}
$$

The last inequality can be easily verified to be true if $A \geq k_{-} /\left(k_{+}+k_{-}\right)$.

(3) This follows from (5.1) and a series of straight forward calculations.

We remark that, if $k_{ \pm} \rightarrow \infty$, the estimate $A \geq 1 / 2$ for the thermodynamic case is recovered, and the large $k$ expansion (5.2) becomes (4.2) with $\rho_{e} \gamma=\mu$.

Proposition 5.2 Let $t \in\left[0, t_{\infty}\right)$.

(1) Let $\mu>0$ and $\nu \geq 0$. We have $\partial_{t} \omega(k, t)<0$ for any wavenumber $k$, if

$$
A(t) \leq\left(\frac{6 \mu D}{F R_{\infty}^{3}}\right)^{2} .
$$

(2) Let $\mu \geq 0$ and $\nu>0$. We have $\partial_{t} \omega(k, t)<0$ for any wavenumber $k$, if

$$
A(t) \leq\left(\frac{24 D \nu}{F k_{-} R_{\infty}^{5}}\right)^{2 / 3} .
$$


Proof. By Part (1) of Proposition 5.1, we may assume that $k \geq 2$.

(1) As in the proof of Proposition 5.1 (2), we get by (5.1) and (5.3) that

$$
\begin{aligned}
\partial_{t} \omega(k, t)=- & \frac{F}{2 A}-\frac{D k\left(\frac{F}{2}+\frac{k_{+} F R_{\infty} A^{1 / 2}}{2 D}+\frac{k_{+} \mu\left(k^{2}-1\right)}{R_{\infty}^{2} A}\right)}{D k+k_{+} R_{\infty} A^{1 / 2}} \\
& +\frac{D k\left(1-A^{k}\right)\left(\frac{F}{2}\left(1+\frac{1}{A}\right)+\frac{k_{-} F R_{\infty} A^{1 / 2}}{2 D}\left(\frac{1}{A}-1\right)-\frac{k_{-} \mu\left(k^{2}-1\right)}{R_{\infty}^{2} A}\right)}{k_{-} R_{\infty} A^{1 / 2}\left(A^{k}+1\right)+D k\left(1-A^{k}\right)} \\
=- & \frac{F}{2 A}-\frac{D k\left(\frac{F}{2}\right)}{D k+k_{+} R_{\infty} A^{1 / 2}}+\frac{D k\left(1-A^{k}\right)\left(\frac{F}{2}\left(1+\frac{1}{A}\right)\right)}{k_{-} R_{\infty} A^{1 / 2}\left(1+A^{k}\right)+D k\left(1-A^{k}\right)} \\
& +\frac{D k\left(1-A^{k}\right)\left(\frac{k_{-} F R_{\infty} A^{1 / 2}}{2 D}\left(\frac{1}{A}-1\right)-\frac{k_{-} \mu\left(k^{2}-1\right)}{R_{\infty}^{2} A}\right)}{k_{-} R_{\infty} A^{1 / 2}\left(1+A^{k}\right)+D k\left(1-A^{k}\right)} \\
<- & \frac{F}{2 A}-\frac{D k\left(\frac{F}{2}\right)}{D k+k_{+} R_{\infty} A^{1 / 2}}+\frac{D k\left(\frac{F}{2}\left(1+\frac{1}{A}\right)\right)}{D k+k_{+} R_{\infty} A^{1 / 2}} \\
& +\frac{D k\left(1-A^{k}\right)\left(\frac{k_{-} F R_{\infty} A^{1 / 2}}{2 D}\left(\frac{1}{A}-1\right)-\frac{k_{-} \mu\left(k^{2}-1\right)}{R_{\infty}^{2} A}\right)}{k_{-} R_{\infty} A^{1 / 2}\left(1+A^{k}\right)+D k\left(1-A^{k}\right)} \\
=- & \frac{F}{2 A}+\frac{D k\left(\frac{F}{2 A}\right)}{D k+k_{+} R_{\infty} A^{1 / 2}}+\frac{D k\left(1-A^{k}\right)\left(\frac{k_{-} F R_{\infty} A^{1 / 2}}{2 D}\right.}{\left.k_{-} R_{\infty} A^{1 / 2}\left(1+A^{k}\right)+D k\left(1-A^{k}\right)-\frac{1}{R_{\infty} A\left(k^{2}-1\right)}\right)} \\
< & \frac{D k\left(1-A^{k}\right)\left(\frac{k_{-} F R_{\infty} A^{1 / 2}}{2 D}\left(\frac{1}{A}-1\right)-\frac{k_{-} \mu\left(k^{2}-1\right)}{R_{\infty}^{2} A}\right)}{k_{-} R_{\infty} A^{1 / 2}\left(1+A^{k}\right)+D k\left(1-A^{k}\right)} \\
\leq & \frac{D k\left(1-A^{k}\right)\left(\frac{k_{-} F R_{\infty} A^{1 / 2}}{2 D A}-\frac{3 k_{-} \mu}{R_{\infty}^{2} A}\right)}{k_{-} R_{\infty} A^{1 / 2}\left(1+A^{k}\right)+D k\left(1-A^{k}\right)} \\
\leq & 0 .
\end{aligned}
$$

(2) By (5.1), we have

$$
\begin{aligned}
\partial_{t} \omega(k, t) \leq- & \frac{F}{2 A}-\frac{\nu\left(k^{4}-k^{2}\right)}{R_{\infty}^{4} A^{2}}-\frac{\frac{F}{2}\left(D k+k k_{+} R_{\infty} A^{1 / 2}\right)}{D k+k_{+} R_{\infty} A^{1 / 2}} \\
& +\frac{D k\left(\frac{F}{2}\left(1+\frac{1}{A}\right)+\frac{k_{-} F R_{\infty} A^{1 / 2}}{2 D}\left(\frac{1}{A}-1\right)\right)}{D k+k_{-} R_{\infty} A^{1 / 2}} \\
< & -\frac{F}{2 A}-\frac{\nu\left(k^{4}-k^{2}\right)}{R_{\infty}^{4} A^{2}}-\frac{F}{2}+\frac{\frac{F}{2}\left(D k\left(\frac{1}{A}+1\right)+k k_{-} R_{\infty} A^{1 / 2}\left(\frac{1}{A}-1\right)\right)}{D k+k_{-} R_{\infty} A^{1 / 2}} \\
<- & \frac{F}{2 A}-\frac{\nu\left(k^{4}-k^{2}\right)}{R_{\infty}^{4} A^{2}}-\frac{F}{2}+\frac{F}{2}\left(\frac{1}{A}+1\right)+\frac{\frac{F}{2} k k_{-} R_{\infty} A^{1 / 2}\left(\frac{1}{A}-1\right)}{D k+k_{-} R_{\infty} A^{1 / 2}} \\
< & -\frac{12 \nu}{R_{\infty}^{4} A^{2}}+\frac{F k_{-} R_{\infty}}{2 D A^{1 / 2}}
\end{aligned}
$$




\section{Q.E.D.}

Remark 5.1 Assume $\mu=\nu=0$. Let $t \in\left[0, t_{\infty}\right)$ be such that $A_{0} \leq A=A(t)<$ $k_{-} /\left(k_{-}+k_{+}\right)$. Then our numerical calculations strongly suggest that there exists a critical value $k_{c}=k_{c}(A)$ such that

$$
\partial_{t} \omega(k, t)<0 \quad \text { for } k<k_{c} \quad \text { and } \quad \partial_{t} \omega(k, t)>0 \quad \text { for } k>k_{c} \text {. }
$$

Figure 5 is a stability diagram showing qualitatively such a critical value for the kinetic boundary condition with $\mu=\nu=0$. The curve, $k=k_{c}(A)$, is obtained by solving the equation $\partial_{t} \omega(k, t)=0$ in which the growth rate $\partial_{t} \omega(k, t)$ is given by $(5.1)$ with $\mu=\nu=0$. The rates are $k_{+}=1$ and $k_{-}=2$ so that $k_{-} /\left(k_{+}+k_{-}\right)=2 / 3$.

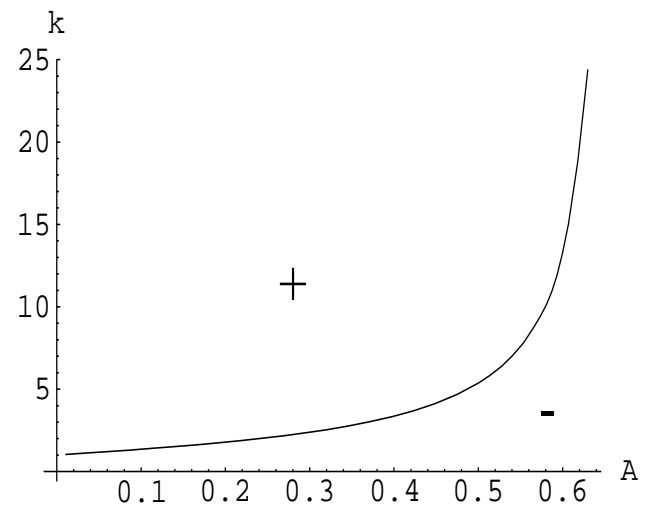

Figure 5: The stability diagram in the $(A, k)$-plane for the kinetic boundary condition with $\mu=\nu=0$ but $F \neq 0$. The curve, $k=k_{c}(A)$, separates the stable region (marked by "-") and the unstable region (marked by "+").

We see from our analysis that the circular island is always stable if its normalized area $A \geq k_{-} /\left(k_{-}+k_{+}\right)$. The island is asymptotically stable, with a decay rate proportional to

$k^{4}$ if the surface diffusion is included, or to $k^{2}$ if the surface diffusion is not but the line tension is included. In general, a small circular island is always stable, if the line tension or surface diffusion is included. If $A<k_{-} /\left(k_{-}+k_{+}\right)$, and if neither the surface diffusion nor line tension is included, then the numerical solution suggests that there exists a critical wavenumber $k_{c}=k_{c}(A)$ such that the island is stable only for wavenumbers $k<k_{c}$.

\section{Conclusions}

We have rigorously analyzed the linear, morphological stability of a single, epitaxially growing, circular island with a radially symmetric adatom distribution, with respect to 
the perturbation in both radial and angular directions. A BCF type island dynamics model that we use consists of the quasi-steady diffusion equation,

$$
-D \Delta \rho=F \quad \text { in } \Omega_{+}(t) \cup \Omega_{-}(t),
$$

a thermodynamics boundary condition (TBC) or a kinetic boundary condition (KBC),

$$
\begin{array}{ll}
\text { TBC: } & \rho=\rho_{e}(1+\gamma \kappa) \quad \text { on } \Gamma(t), \\
\text { KBC: } \quad\left\{\begin{array}{r}
-D \nabla \rho_{+} \cdot n=k_{+}\left(\rho_{+}-\rho_{*}-\mu \kappa\right) \\
D \nabla \rho_{-} \cdot n=k_{-}\left(\rho_{-}-\rho_{*}-\mu \kappa\right)
\end{array} \quad \text { on } \Gamma(t),\right.
\end{array}
$$

the normal velocity

$$
v=-D[\nabla \rho \cdot n]+\nu \kappa_{s s}
$$

the continuity of the adatom density at the center of island, and the flux-free far-field condition.

We summarize in Table 6.1 our analysis on the asymptotic behavior of the growth rate

\begin{tabular}{|c|c|}
\hline BCs and velocity & $\partial_{t} \omega(k, t)$ as $k \rightarrow \infty$ \\
\hline TBC $\gamma=\nu=0$ & $\begin{array}{l}\frac{F(1-2 A)}{2 A} k+O(1) \\
\text { asymptotically unstable if } A<1 / 2\end{array}$ \\
\hline TBC $\gamma \neq 0$ or $\nu \neq 0$ & $\begin{array}{l}-\tilde{\nu} k^{4}-2 \tilde{\gamma} k^{3}+O\left(k^{2}\right) \\
\text { asymptotically stable }\end{array}$ \\
\hline $\mathrm{KBC} \mu=\nu=0$ & $\begin{array}{l}\frac{F R_{\infty} A^{1 / 2}}{2 D}\left(k_{-}\left(\frac{1}{A}-1\right)-k_{+}\right)+O\left(\frac{1}{k}\right) \\
\text { asymptotically unstable if } A<k_{-} /\left(k_{+}+k_{-}\right)\end{array}$ \\
\hline KBC $\mu \neq 0$ or $\nu \neq 0$ & $\begin{array}{l}-\tilde{\nu} k^{4}+\left(\tilde{\nu}-\left(k_{+}+k_{-}\right) \tilde{\mu}\right) k^{2}+O(k) \\
\text { asymptotically stable }\end{array}$ \\
\hline
\end{tabular}
$\partial_{t} \omega(k, t)$ and the corresponding asymptotic stability or instability for large wavenumbers. For convenience, we use the notation

$$
\tilde{\nu}=\frac{\nu}{R_{\infty}^{4} A^{2}}, \quad \tilde{\gamma}=\frac{D \rho_{e} \gamma}{R_{\infty}^{3} A^{3 / 2}}, \quad \tilde{\mu}=\frac{\mu}{R_{\infty}^{2} A} .
$$

Table 6.1. A summary of the asymptotic stability analysis.

We make the following concluding remarks:

(1) For both the thermodynamic and kinetic boundary conditions, a circular island is always asymptotically stable, if the line tension or surface diffusion is included;

(2) For both the thermodynamic and kinetic boundary conditions, a small island is stable for all wavenumbers, if the line tension or surface diffusion is included;

(3) For the thermodynamic boundary condition, a circular island is always stable, if its normalized area $A \geq 1 / 2$; 
(4) For the kinetic boundary condition, a circular island is always stable, if its normalized area $A \geq k_{-} /\left(k_{-}+k_{+}\right)$;

(5) For the thermodynamic boundary condition, if the line tension and surface diffusion are absent, and if the normalized area $A \leq 1 / 2$, then there exists a unique critical wavenumber $k_{c}=k_{c}(A)$ such that the circular island is stable for any wavenumber $k<k_{c}$ and unstable for any $k>k_{c}$;

(6) For the kinetic boundary condition, if the line tension and surface diffusion are absent, and if the normalized area $A \leq k_{-} /\left(k_{-}+k_{+}\right)$, then there exists a unique critical wavenumber $k_{c}=k_{c}(A)$ such that the circular island is stable for any wavenumber $k<k_{c}$ and unstable for any $k>k_{c}$;

(7) The Bales-Zangwill instability for straight atomic steps due to the Ehrlich-Schwoebel effect disappears for small circular islands, if either the line tension or surface diffusion is included.

Acknowledgments. The authors thank Dr. Michael Moske, Dr. Peter Smereka, and Dr. John Weeks for helpful discussions. They also thank Dr. John Weeks for bringing to their attention the reference [23].

\section{Appendix}

We derive the boundary condition (3.22) and (3.23), and the velocity (3.21) for the linearized system (3.17)-(3.23). First, consider a curve $\Gamma_{0}: r=r_{0}(\theta)$ in the polar coordinate system. It can be expressed as

$$
x=r_{0}(\theta) \cos \theta, \quad y=r_{0}(\theta) \sin \theta
$$

in the Cartesian coordinate system. Here we use $(x, y)$ instead of $\left(x_{1}, x_{2}\right)$ to denote a generic point. The unit exterior normal $n_{0}$ and the curvature $\kappa_{0}$ of this curve are defined by

$$
\begin{aligned}
& n_{0}=\frac{\left(y_{\theta},-x_{\theta}\right)}{\sqrt{x_{\theta}^{2}+y_{\theta}^{2}}}=\frac{r_{0}^{\prime}(\theta)(\sin \theta,-\cos \theta)+r_{0}(\theta)(\cos \theta, \sin \theta)}{\sqrt{\left(r_{0}(\theta)\right)^{2}+\left(r_{0}^{\prime}(\theta)\right)^{2}}}, \\
& \kappa_{0}=\frac{x_{\theta} y_{\theta \theta}-x_{\theta \theta} y_{\theta}}{\left(x_{\theta}^{2}+y_{\theta}^{2}\right)^{3 / 2}}=\frac{\left(r_{0}(\theta)\right)^{2}+2\left(r_{0}^{\prime}(\theta)\right)^{2}-r_{0}(\theta) r_{0}^{\prime \prime}(\theta)}{\left(\left(r_{0}(\theta)\right)^{2}+\left(r_{0}^{\prime}(\theta)\right)^{2}\right)^{3 / 2}},
\end{aligned}
$$

where a subscript denotes a derivative. The tangential derivative of a smooth function $h=h(\theta)$ defined on $\Gamma_{0}$ is

$$
\frac{d h(\theta)}{d s}=\frac{h^{\prime}(\theta)}{\sqrt{x_{\theta}^{2}+y_{\theta}^{2}}}=\frac{h^{\prime}(\theta)}{\sqrt{\left(r_{0}(\theta)\right)^{2}+\left(r_{0}^{\prime}(\theta)\right)^{2}}} .
$$


Since the curve $\tilde{\Gamma}(t)$ is given by $r=R(t)+\varepsilon R_{1}(\theta, t)$, we obtain by (A.1), (A.2), and the Taylor expansion that

$$
\begin{aligned}
\tilde{n} & =\frac{\varepsilon \partial_{\theta} R_{1}(\theta, t)(\sin \theta,-\cos \theta)+\left(R(t)+\varepsilon R_{1}(\theta, t)\right)(\cos \theta, \sin \theta)}{\sqrt{\left(\varepsilon \partial_{\theta} R_{1}(\theta, t)\right)^{2}+\left(R(t)+\varepsilon R_{1}(\theta, t)\right)^{2}}} \\
& =n+\varepsilon n_{1}+O\left(\varepsilon^{2}\right), \\
\tilde{\kappa} & =\frac{\left(R(t)+\varepsilon R_{1}(\theta, t)\right)^{2}+2\left(\varepsilon \partial_{\theta} R_{1}(\theta, t)\right)^{2}-\left(R(t)+\varepsilon R_{1}(\theta, t)\right)\left(\varepsilon \partial_{\theta \theta} R_{1}(\theta, t)\right)}{\left\{\left(R(t)+\varepsilon R_{1}(\theta, t)\right)^{2}+\left(\varepsilon \partial_{\theta} R_{1}(\theta, t)\right)^{2}\right\}^{3 / 2}} \\
& =\kappa+\varepsilon \kappa_{1}+O\left(\varepsilon^{2}\right),
\end{aligned}
$$

where $n$ and $\kappa$ are given in (3.1), and

$$
\begin{aligned}
n_{1} & =\frac{\partial_{\theta} R_{1}(\theta, t)}{R(t)}(\sin \theta,-\cos \theta), \\
\kappa_{1} & =-\frac{1}{R(t)^{2}}\left(\partial_{\theta \theta} R_{1}(\theta, t)+R_{1}(\theta, t)\right) .
\end{aligned}
$$

Now, we have by (3.12) that

$$
\begin{aligned}
\left.\tilde{\rho}_{ \pm}(r, \theta, t)\right|_{\tilde{\Gamma}(t)} & =\rho_{ \pm}\left(R(t)+\varepsilon R_{1}(\theta, t)\right)+\varepsilon \rho_{1 \pm}\left(R(t)+\varepsilon R_{1}(\theta, t), \theta, t\right) \\
& =\rho_{ \pm}(R(t), t)+\varepsilon\left(R_{1}(\theta, t) \partial_{r} \rho_{ \pm}(R(t), t)+\rho_{1 \pm}(R(t), \theta, t)\right)+O\left(\varepsilon^{2}\right) .
\end{aligned}
$$

Similarly, we have by (3.12) and (A.4) that

$$
\begin{aligned}
\nabla \tilde{\rho}_{ \pm} & \left.(r, \theta, t) \cdot \tilde{n}\right|_{\tilde{\Gamma}(t)} \\
= & \left\{\nabla \rho_{ \pm}\left(R(t)+\varepsilon R_{1}(\theta, t), t\right)+\varepsilon \nabla \rho_{1 \pm}\left(R(t)+\varepsilon R_{1}(\theta, t), \theta, t\right)\right\} \cdot\left(n+\varepsilon n_{1}+O\left(\varepsilon^{2}\right)\right) \\
= & \left\{\nabla \rho_{ \pm}(R(t), t)+\varepsilon R_{1}(\theta, t) \nabla \partial_{r} \rho_{ \pm}(R(t), t)+\varepsilon \nabla \rho_{1 \pm}(R(t), \theta, t)\right\} \cdot\left(n+\varepsilon n_{1}\right)+O\left(\varepsilon^{2}\right) \\
= & \nabla \rho_{ \pm}(R(t), t) \cdot n+\varepsilon \nabla \rho_{ \pm}(R(t), t) \cdot n_{1} \\
& \quad+\varepsilon R_{1}(\theta, t) \nabla \partial_{r} \rho_{ \pm}(R(t), t) \cdot n+\varepsilon \nabla \rho_{1 \pm}(R(t), \theta, t) \cdot n+O\left(\varepsilon^{2}\right) .
\end{aligned}
$$

Note for any radially symmetric function $g=g(r)$ that $\nabla g(r)=g^{\prime}(r)(\cos \theta, \sin \theta)$. Thus, by (3.1) and (A.6), $\nabla g(r) \cdot n=g^{\prime}(r)$ and $\nabla g(r) \cdot n_{1}=0$. Consequently,

$$
\begin{aligned}
& \left.\nabla \tilde{\rho}_{ \pm}(r, \theta, t) \cdot \tilde{n}\right|_{\tilde{\Gamma}(t)} \\
& \quad=\nabla \rho_{ \pm}(R(t), t) \cdot n+\varepsilon\left(R_{1}(\theta, t) \partial_{r r} \rho_{ \pm}(R(t), t)+\partial_{r} \rho_{1 \pm}(R(t), \theta, t)\right)+O\left(\varepsilon^{2}\right) .
\end{aligned}
$$

Inserting (A.8) and (A.9) into (3.14) and (3.15), using (2.2), (2.3), (A.5), and (A.7), and comparing terms of order $\varepsilon$, we obtain the thermodynamic and kinetic boundary conditions (3.22) and (3.23) for $\rho_{1}$.

By (3.13), the velocity of the boundary $\tilde{\Gamma}(t): r=R(t)+\varepsilon R_{1}(\theta, t)$ is

$$
\tilde{v}=R^{\prime}(t)+\varepsilon \partial_{t} R_{1}(\theta, t)
$$


Moreover, by (A.3), (A.5), (A.7), and (3.1),

$$
\tilde{\kappa}_{\tilde{s} \tilde{s}}=-\frac{\varepsilon}{(R(t))^{4}}\left(\partial_{\theta \theta \theta \theta} R_{1}(\theta, t)+\partial_{\theta \theta} R_{1}(\theta, t)\right)+O\left(\varepsilon^{2}\right) .
$$

Now, inserting (A.9)-(A.11) into (3.16), and using (3.6) and the fact that $\kappa_{s s}=0$, we obtain the velocity formula (3.21) from the $O(\varepsilon)$ terms.

\section{References}

[1] M. Avignon. Role of interface kinetics on the shape stability of a two-dimensional nucleus on a substrate. J. Crys. Growth, 11:265-272, 1971.

[2] M. Avignon. Shape tability of a two-dimensional nucleus. J. Crys. Growth, 13/14:113-120, 1972.

[3] G. S. Bales and A. Zangwill. Morphological instability of a terrace edge during step-flow growth. Phys. Rev. B, 41(9):5500-5508, 1990.

[4] A.-L. Barabási and H. E. Stanley. Fractal Concepts in Surface Growth. Cambridge University Press, Cambridge, 1995.

[5] W. K. Burton, N. Cabrera, and F. C. Frank. The growth of crystals and the equilibrium of their surfaces. Phil. Trans. Roy. Soc. London Ser. A, 243(866):299-358, 1951.

[6] R. E. Caflisch, W. E, M. F. Gyure, B. Merriman, and C. Ratsch. Kinetic model for a step edge in epitaxial growth. Phys. Rev. E, 59(6):6879-6887, 1999.

[7] R. E. Caflisch and B. Li. Analysis of island dynamics in epitaxial growth of thin films. Multiscale Model. and Anal., 1:150-171, 2003.

[8] G. Ehrlich and F. G. Hudda. Atomic view of surface diffusion: tungsten on tungsten. J. Chem. Phys., 44:1036-1099, 1966.

[9] T. Fujioka and R. F. Sekerka. Morphological stability of disk crystals. J. Crys. Growth, 24/25:84-93, 1974.

[10] R. Ghez, H. G. Cohen, and J. B. Keller. The stability of growing or evaporating crystals. J. Appl. Phys., 73:3685-3693, 1993.

[11] R. Ghez and S. S. Iyer. The kinetics of fast steps on crystal surfaces and its application to the molecular beam epitaxy of silicon. IBM J. Res. Develop., 32:804-818, 1988.

[12] A. Karma and C. Misbah. Competition between noise and determinism in step flow growth. Phys. Rev. Lett., 71:3810-3813, 1993. 
[13] J. B. Keller, H. G. Cohen, and G. J. Merchant. The stability of rapidly growing crystals. J. Appl. Phys., 73:3694-3697, 1993.

[14] J. Krug. Four lectures on the physics of crystal growth. Physica A, 313:47-82, 2002.

[15] F. Liu and H. Metiu. Stability and kinetics of step motion on crystal surfaces. Phys. Rev. E, 49:2601-2616, 1997.

[16] K. Morgenstern, G. Rosenfeld, and G. Comsa. Decay of two-dimensional Ag islands on $\operatorname{Ag}(111)$. Phys. Rev. Lett., 76(12):2113-2116, 1996.

[17] O. Pierre-Louis. Continuum model for low temperature relaxation of crystal shapes. Phys. Rev. Lett., 87:106104-1-4, 2001.

[18] A. Pimpinelli and J. Villain. Physics of Crystal Growth. Cambridge University Press, Cambridge, 1998.

[19] P. Politi, G. Grenet, A. Marty, A. Ponchet, and J. Villain. Instabilities in crystal growth by atomic or molecular beams. Physics Reports, 324:271-404, 2000.

[20] R. L. Schwoebel. Step motion on crystal surfaces II. J. Appl. Phys., 40:614-618, 1969.

[21] R. L. Schwoebel and E. J. Shipsey. Step motion on crystal surfaces. J. Appl. Phys., 37:3682-3686, 1966.

[22] W. Theis, N. C. Bartelt, and R. M. Tromp. Chemical potential maps and spatial correlations in 2D-island ripening on $\mathrm{Si}(001)$. Phys. Rev. Lett., 75(18):3328-3331, 1995.

[23] K. Thürmer, E. Williams, and J. Reutt-Robey. Autocatalytic oxidation of lead crystallite surfaces. Science, 297:2033-2035, 2002. 